\title{
Ruptured pediatric cerebellopontine angle epidermoid cyst: a case report detailing radiographic evolution and clinical course
}

\author{
*Zhe Guan, MS,, Todd Hollon, MD, ${ }^{2}$ J. Nicole Bentley, MD, ${ }^{2}$ and Hugh J. L. Garton, MD, MHSc ${ }^{2}$ \\ 1 University of Michigan Medical School; and 2Department of Neurosurgery, University of Michigan Health System, Ann Arbor, \\ Michigan
}

\begin{abstract}
Epidermoid cysts (ECs) are uncommon pediatric tumors that often occur in the cerebellopontine angle. Although cyst rupture is a recognized complication, the radiographic evolution of an EC following rupture and the resultant parenchymal brainstem edema have not been reported. The authors present the case of a 13-year-old female with a newly diagnosed cerebellopontine angle EC who presented with worsening headaches, photophobia, and emesis. Magnetic resonance imaging demonstrated significant pericystic brainstem edema and mass effect with effacement of the fourth ventricle. Refractory symptoms prompted repeat imaging, revealing cyst enlargement and dense rim enhancement. Resection of the EC resolved both her symptoms and the brainstem edema. This case documents the radiographic evolution of EC rupture and subsequent clinical course.
\end{abstract}

http://thejns.org/doi/abs/10.3171/2015.4.PEDS153

KEY WORDS cerebellopontine angle tumor; chemical meningitis; cyst rupture; epidermoid cyst; oncology

$\mathrm{E}$ PIDERMOID cysts (ECs) represent about $1 \%$ of all intracranial tumors. ${ }^{19,28}$ Their incidence is highest in the 3rd to 5th decades of life, and they infrequently occur in pediatric patients. Intracranial ECs are benign congenital tumors that arise from displaced ectoderm during neural tube closure early in embryonic development. ${ }^{6,19,28}$ About $40 \%-50 \%$ of intracranial ECs are found in the cerebellopontine angle (CPA), with other common sites including the parasellar region, along the tentorium, and the fourth ventricle. ${ }^{6,10,23}$ Epidermoid cysts constitute $4 \%$ of all tumors found in the CPA, making it the third most common CPA tumor behind vestibular schwannomas and meningiomas in children and adults. ${ }^{11}$ In contrast, dermoid cysts, although similar in pathophysiology with the inclusion of ectopic ectoderm during neural tube closure, are otherwise easily differentiated from ECs by their midline location, radiographic findings consistent with fat rather than CSF, and microscopic features. Additionally, dermoid cysts compose only $0.5 \%$ of all intracranial tumors, making them less common.

The natural history of ECs is that of indolent linear growth beginning at birth, with symptoms developing later in life because of neurovascular or brainstem compression. Chronic symptoms such as headache, cranial neuropathies, and cerebellar dysfunction are often present for months to years prior to diagnosis. ${ }^{12,18,20,21,24}$ Additionally, following cyst rupture, patients may present acutely with aseptic chemical meningitis, also known as Mollaret's meningitis. ${ }^{8}$

The literature contains several reported cases of EC rupture heralded by meningismus and CSF analysis consistent with chemical meningitis. ${ }^{1,4,8,22}$ However, the correlation of these clinical findings with radiographic evidence of rupture has not been reported. Here, we present the case of a 13-year-old female presenting with CPA EC rupture resulting in significant pericystic brainstem edema as seen on MRI. We review her subsequent clinical course and operative intervention. The current literature on the management of pediatric ECs is also summarized.

\section{Case Report}

Clinical Presentation

A 13-year-old right-handed female with a history of

ABBREVIATIONS $\mathrm{CN}=$ cranial nerve; $\mathrm{CPA}=$ cerebellopontine angle; $\mathrm{EC}=$ epidermoid cyst; $\mathrm{ED}=$ emergency department . SUBMITTED January 2, 2015. ACCEPTED April 16, 2015.

* Mr. Guan and Dr. Hollon contributed equally to this work. 
headaches presented to the pediatric emergency department (ED) with a 3-day severe, atypical occipital headache associated with dizziness. At this time she denied photophobia, emesis, or neck stiffness and had a normal neurological exam. She was initially treated with ketorolac, prochlorperazine, and diphenhydramine and was discharged from the ED with a planned outpatient follow-up with her pediatrician.

Three days later, she returned to the ED with continued severe atypical pain, reporting that the medications provided only transient relief. An MRI study was performed, revealing a $2.8 \times 1.3 \times 2.5-\mathrm{cm}$ nonenhancing lesion in the right CPA exerting mild mass effect on the adjacent pons. The lesion was characterized by minimal pericystic FLAIR signal (Fig. 1A) or rim enhancement (Fig. 1C), with densely restricted diffusion (Fig. 1B). Given these imaging findings, the patient was diagnosed with an EC. Because she had no focal neurological signs referable to the mass lesion and because MRI was not believed to show an acute process, a migraine headache syndrome was considered to be a likely etiology of her symptoms. The patient was conservatively managed with analgesics and discharged with a recommendation for outpatient follow-up.

Approximately 2 weeks later, she again presented to the ED with refractory symptoms now associated with nausea, emesis, and photophobia, and methylprednisolone was prescribed. Treatment initially relieved her symptoms, but she returned 1 week later with recurrent headaches and nausea, and dihydroergotamine was administered intravenously. Because of the limited response to medi-
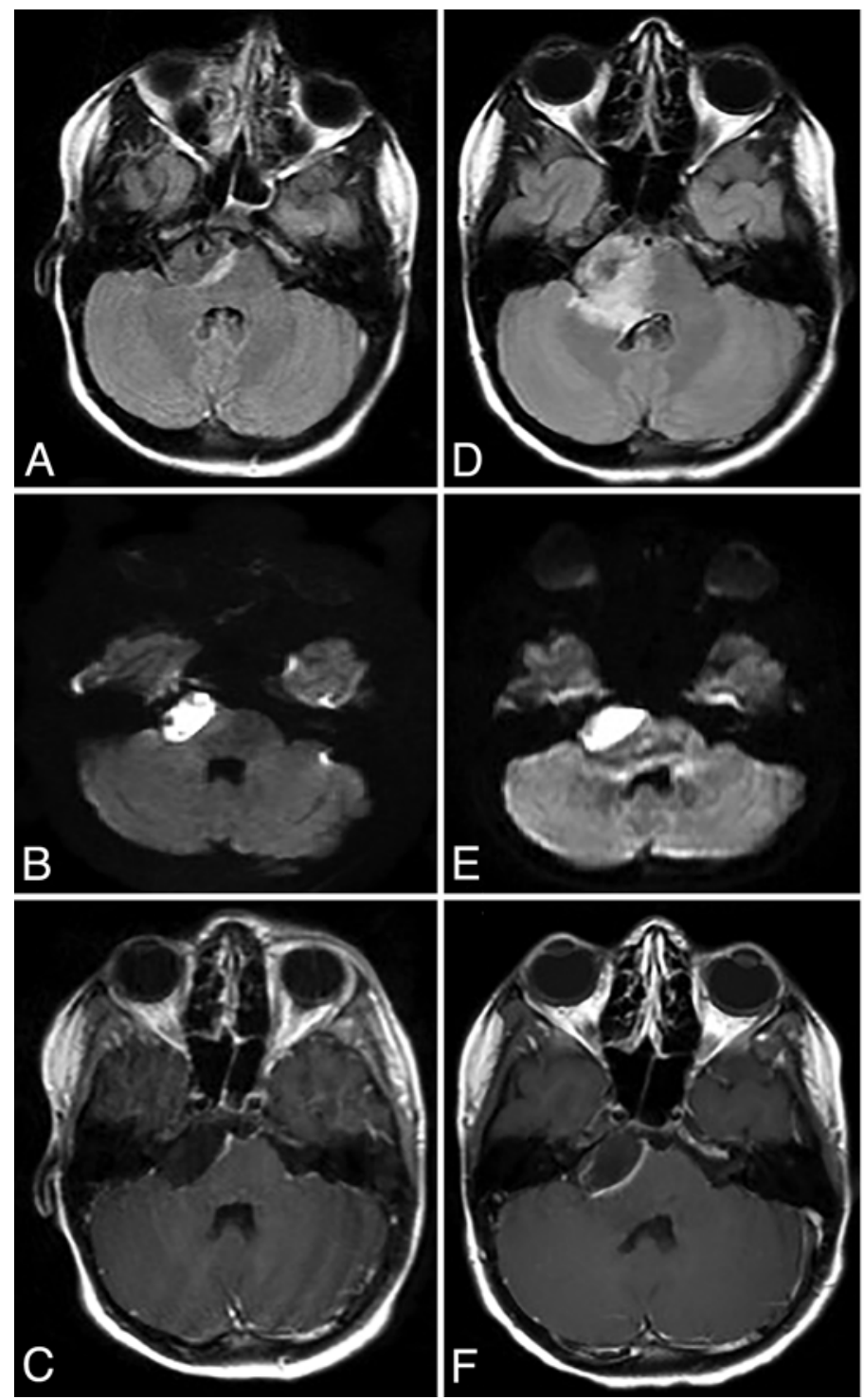
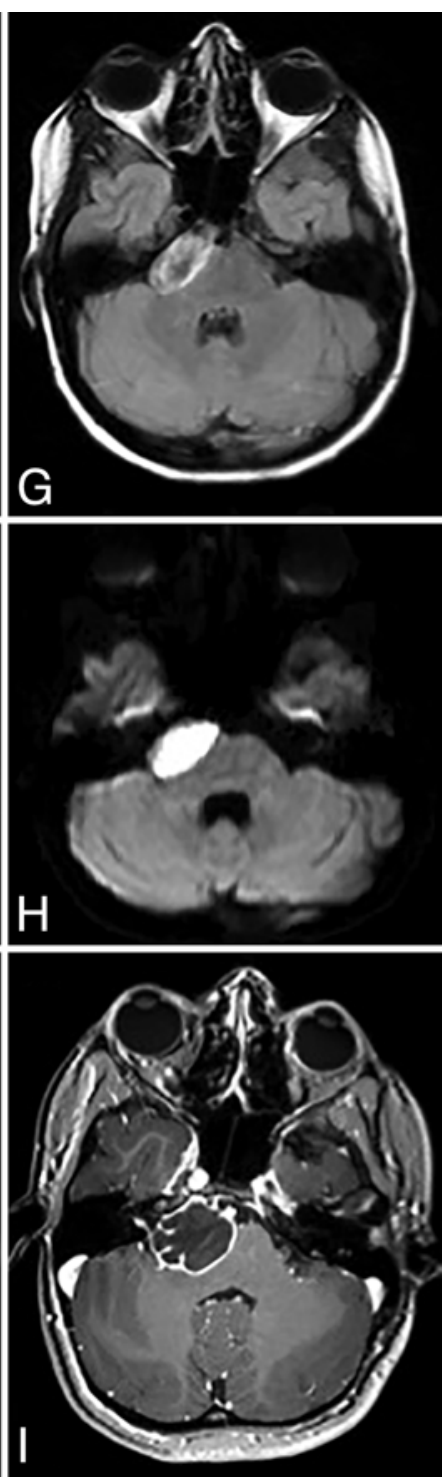

FIG. 1. Brain FLAIR (upper), diffusion-weighted (center), and T1-weighted (lower) post-Gd sequences demonstrating the progression of brainstem edema and cyst growth following EC rupture. A-C: Initial imaging shows the classic appearance of a CPA EC with mild compression of adjacent brainstem and dense diffusion restriction. D-F: Following cyst rupture, parenchymal edema with mass effect is seen involving the pons and cerebellum leading to distortion of the fourth ventricle. G-I: Resolution of edema with the administration of dexamethasone is seen on FLAIR. Note that the image in panel I is a preoperative MRI study for stereotactic navigation completed approximately 3 weeks after the images in panels $\mathrm{G}$ and $\mathrm{H}$. 
cal therapy, her diagnosis was reconsidered and a lumbar puncture was performed. This revealed an opening pressure of $31 \mathrm{~mm} \mathrm{Hg}$, a white blood cell count of 240/ $\mathrm{\mu l}(50 \%$ lymphocytes, $41 \%$ neutrophils), a red blood cell count of 3/ $\mu l$, protein level of $94 \mathrm{mg} / \mathrm{dl}$, and glucose level of $55 \mathrm{mg} /$ dl. Repeat MRI demonstrated extensive new FLAIR signal abnormalities within the pons and middle cerebellar peduncle, with extension into the right cerebellar hemisphere (Fig. 1D). The lesion was not significantly changed in size and continued to exert mass effect, with distortion of the fourth ventricle. A new thickened enhancing rim suggesting reactive changes was present (Fig. 1F). In view of these clinical findings, EC rupture and chemical meningitis were diagnosed. Since the patient was neurologically intact, she was given a trial of dexamethasone that led to the rapid resolution of her symptoms. She was discharged on hospital Day 5 with a planned outpatient follow-up.

Two weeks later, she returned for an outpatient followup, reporting good control of her symptoms. At that time, repeat MRI showed radiographic resolution of the brainstem edema (Fig. 1G). A long steroid taper was initiated but resulted in recurrent headaches and dizziness with accompanying eye discomfort and emesis. Because the EC had not spontaneously resolved and given the intolerable side effects of the steroids, the decision was made to proceed with operative intervention. A thin-slice MRI study was performed for intraoperative navigation and showed significant interval enlargement $(2.7 \times 2.7 \times 3.4 \mathrm{~cm})$ with an avidly contrast-enhancing rim (Fig. $1 \mathrm{H}$ and I). Nearcomplete effacement of the fourth ventricle was noted in response to increased mass effect from the expanding EC following rupture.

\section{Operative Intervention and Pathological Findings}

A right retrosigmoid craniotomy was performed, and a pearly white lesion was found deep to and spanning the cranial nerve $(\mathrm{CN})$ complexes of VII-VIII and IX-XI (Fig. 2). From the viewable operative corridor, the capsule of the lesion was not obviously ruptured, and no cyst contents were visibly free in the subarachnoid space. However, only a small portion of the entire capsule was visible. The capsule was opened and caseous material was extracted. Intracapsular gross-total resection was performed, with removal of the tumor in a piecemeal fashion. On inspection, the capsule was quite variable in thickness and strength. As the caseous material was removed, we observed several perforations associated with thin areas of the capsule, although it is possible these were the result of the dissection process. Other portions of the capsule were thicker and densely adherent to the surrounding CNs. These areas were left behind to avoid $\mathrm{CN}$ injury. Histological examination of intraoperative tumor specimens showed a cyst capsule composed of stratified squamous epithelium. Cystic contents demonstrated laminated keratin debris, as well as compact keratin components (Fig. 3).

\section{Postoperative Course}

The patient tolerated the procedure well and was discharged on postoperative Day 3 without any neurological deficits. At the 3-month follow-up, she remained clinically well and had returned to school without any functional
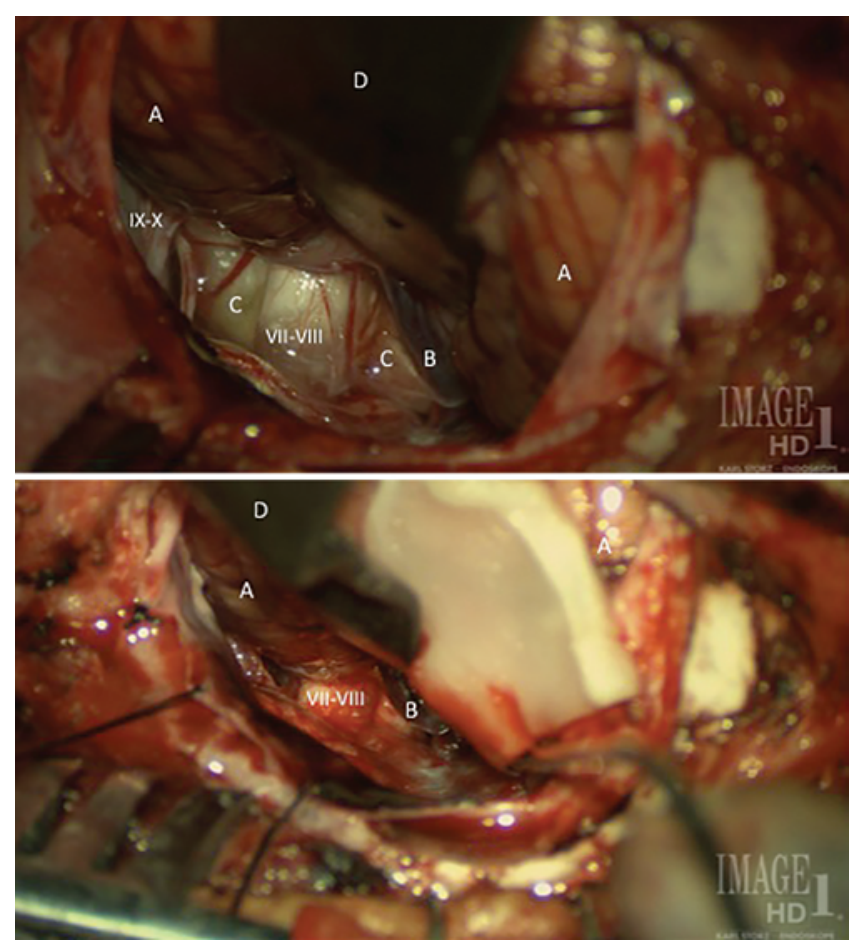

FIG. 2. Intraoperative preresection (upper) and postresection (lower) photographs of the right CPA as viewed through a right retrosigmoid approach. A compressed CN VII-VIII complex is draped over the lateral aspect of the tumor. The CN IX-XI complex can be visualized abutting the inferior aspect of the tumor. $A=$ cerebellum; $B=$ petrosal vein; $C=$ tumor; D = retractor; VII-VIII = CN VII-VIII complex; IX-XI = CN IX-XI complex. Figure is available in color online only.

impairment. She reported only mild headaches that were easily treated with ibuprofen. Follow-up MRI at 6 months revealed resolution of the brainstem edema (Fig. 4A) and a small residual capsule. No rim enhancement was noted, and normal fourth ventricular anatomy had been restored (Fig. 4B and C).

\section{Discussion}

This case is the first description of the clinical course of a ruptured pediatric CPA EC with corresponding radiographic evolution. Although the capsule did not appear to be open on initial operative inspection, we could only visualize a small portion of the total surface of the lesion.
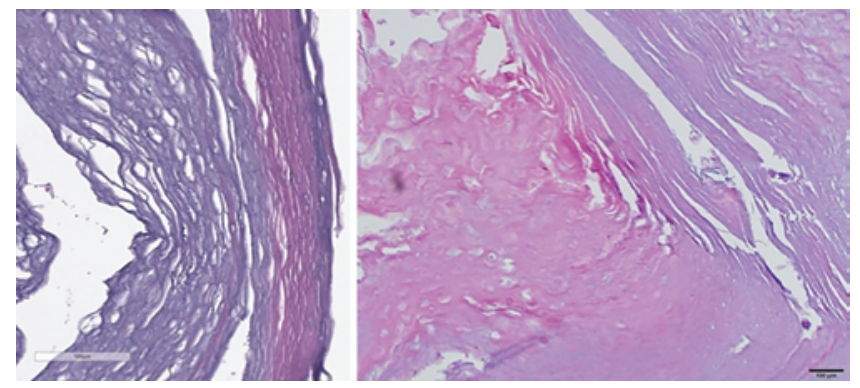

FIG. 3. Intraoperative tumor specimens stained with H \& E. Left: Tumor capsule with keratinized stratified squamous epithelium. Right: Cystic contents containing both laminated and compact keratin. Bar $=100 \mu \mathrm{m}$. Figure is available in color online only. 

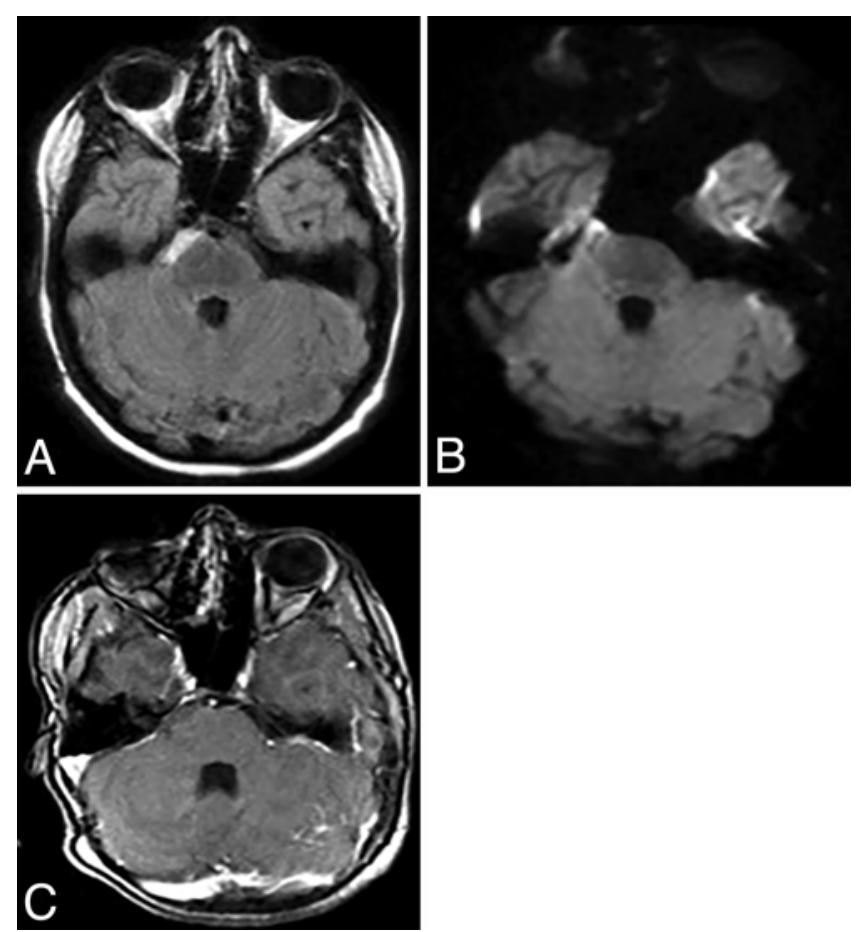

FIG. 4. Postoperative brain FLAIR (A), diffusion-weighted (B), and T1weighted (C) post-Gd sequences at the 6 -month follow-up showing subtotal resection with a small amount of residual cyst medially. No areas of enhancement or mass effect are visible. Note that the fourth ventricle appears normal without distortion.

More ventrally, the cyst wall was observed to be incompetent (not intact, with holes) after the dissection. Additionally, the presence of CSF pleocytosis and elevated protein count, along with the patient's response to and dependence on corticosteroids for symptomatic relief, as well as the MRI findings, argue strongly for exposure of the brainstem and CSF to an inflammatory source, probably the caseous material of the lesion through cyst wall incompetence and rupture. Alternatively, if the cyst wall was fully competent, it follows that it was the primary inflammatory stimulus, rather than the cyst contents. Pathologically, the cyst wall did not appear unusual.

The radiographic characteristics of unruptured ECs have been well described. ${ }^{5,16}$ Contrasted CT shows a well-circumscribed, nonenhancing hypodense mass with variable lobulations, septations, and calcifications. Hyperdense or enhancing tumors are rare. Magnetic resonance imaging demonstrates iso- or hypointense masses on T1-weighted sequences and hyperintense lesions on T2-weighted images. Diffusion-weighted and FLAIR sequences remain invaluable for differentiating ECs from arachnoid cysts. Both have the appearance of CSF on CT and T1- and T2-weighted MRI, though ECs intensely restrict diffusion and do not suppress FLAIR signal. ${ }^{9}$ The traditional description of ECs is that of a well-circumscribed lesion without parenchymal edema despite the presence of significant mass effect. . $^{17,25,26}$ We present this case to illustrate the significant parenchymal edema that can result from cyst rupture and the first description in the literature of a cyst in clinical and radiographic evolution.

The true incidence of cyst rupture is uncertain, but it is thought to be rare, resulting in a clinical presentation consistent with chemical meningitis. ${ }^{15,20}$ Patients with persistent or disabling symptoms related to neurovascular compression or recurrent cyst rupture should be offered surgical intervention. In patients with normal neurological function and no signs of raised intracranial pressure, conservative management with close observation may be recommended initially. Because ECs grow slowly, this approach can make surgery unnecessary or delay surgery by several years. ${ }^{7}$ As these lesions are poorly responsive to chemotherapy and radiation, excision remains the definitive treatment. ${ }^{6}$ Ideally, gross-total resection is performed to minimize the risk of tumor recurrence. The overall recurrence rate is reported to range from $0 \%$ to $36 \%$. . $^{2,20,21,27}$ The rate of reoperation due to recurrence ranges from $0 \%$ to $21 \% .{ }^{21,27}$ The natural history of recurrence in these lesions is difficult to ascertain from the literature, as the relatively short follow-up and slow growth pattern limit proper assessment. ${ }^{21}$ The prospect of recurrence due to postoperative residual cyst presents a difficult challenge for intraoperative decision making, as the tumor capsule is often densely adherent to neighboring neurovascular structures. ${ }^{19,24}$ Controversy exists between those who advocate for maximum resection at the cost of possible neurological deficit to prevent recurrence 3,27 and those who favor a more conservative approach, accepting subtotal resection to avoid both transient and permanent neurological injury. ${ }^{13,20}$ Recent data suggest that the total removal of CPA ECs does not significantly increase morbidity or mortality and should be the operative goal, although another series reported similar rates of recurrence for grosstotal and subtotal resection ( $23 \%$ and $27 \%$, respectively). ${ }^{21}$ Clinical judgment must ultimately be used to determine the most appropriate extent of resection for each patient based on clinical presentation and intraoperative findings.

Regardless of the extent of tumor removal, complications are relatively common. ${ }^{12,21}$ Aseptic meningitis is reported to occur in 10\%-40\% of cases. This is attributed to intraoperative spillage of cyst contents and can usually be treated successfully with a short course of oral steroids..$^{14,20}$ Transient $\mathrm{CN}$ deficits are common following surgery, especially affecting nearby CNs V-VIII. Deficits of CNs VVII and X tend to be transient and typically resolve over $0.5-1.5$ years, but damage to CN VIII with hearing loss often persists. ${ }^{7,14}$ Less common complications include CSF leakage (4\%-8\%), symptomatic hydrocephalus (14\%), vestibular or cerebellar dysfunction, and pseudomeningocele $(5 \%)^{2,3,14,15,21}$

\section{Conclusions}

Following CPA EC rupture causing pericystic brainstem edema, our patient experienced resolution of her presenting symptoms following subtotal resection without postoperative neurological deficits or complications. This case warrants attention because it is the first report to demonstrate that cyst rupture leads to radiographic findings that challenge the traditional description of a mass lesion without significant parenchymal edema. The documentation of a ruptured EC in evolution provides evidence for the possibility of a more insidious clinical course resulting from these "benign" lesions. 


\section{References}

1. Abramson RC, Morawetz RB, Schlitt M: Multiple complications from an intracranial epidermoid cyst: case report and literature review. Neurosurgery 24:574-578, 1989

2. Ahmed I, Auguste KI, Vachhrajani S, Dirks PB, Drake JM, Rutka JT: Neurosurgical management of intracranial epidermoid tumors in children. Clinical article. J Neurosurg Pediatr 4:91-96, 2009

3. Altschuler EM, Jungreis CA, Sekhar LN, Jannetta PJ, Sheptak PE: Operative treatment of intracranial epidermoid cysts and cholesterol granulomas: report of 21 cases. Neurosurgery 26:606-614, 1990

4. Becker WJ, Watters GV, de Chadarevian JP, Vanasse M: Recurrent aseptic meningitis secondary to intracranial epidermoids. Can J Neurol Sci 11:387-389, 1984

5. Chen S, Ikawa F, Kurisu K, Arita K, Takaba J, Kanou Y: Quantitative MR evaluation of intracranial epidermoid tumors by fast fluid-attenuated inversion recovery imaging and echo-planar diffusion-weighted imaging. AJNR Am J Neuroradiol 22:1089-1096, 2001

6. Cobbs CS, Pitts LH, Wilson CB: Epidermoid and dermoid cysts of the posterior fossa. Clin Neurosurg 44:511-528, 1997

7. Darrouzet V, Franco-Vidal V, Hilton M, Nguyen DQ, LacherFougere S, Guerin J, et al: Surgery of cerebellopontine angle epidermoid cysts: role of the widened retrolabyrinthine approach combined with endoscopy. Otolaryngol Head Neck Surg 131:120-125, 2004

8. de Chadarévian JP, Becker WJ: Mollaret's recurrent aseptic meningitis: relationship to epidermoid cysts. Light microscopic and ultrastructural cytological studies of the cerebrospinal fluid. J Neuropathol Exp Neurol 39:661-669, 1980

9. Dutt SN, Mirza S, Chavda SV, Irving RM: Radiologic differentiation of intracranial epidermoids from arachnoid cysts. Otol Neurotol 23:84-92, 2002

10. Fromm H, Huf H, Schaffer M: Operation and results in 102 tumors of the cerebellopontine angle. Adv Neurosurg 1:260-261, 1973

11. Holman MA, Schmitt WR, Carlson ML, Driscoll CL, Beatty CW, Link MJ: Pediatric cerebellopontine angle and internal auditory canal tumors: clinical article. J Neurosurg Pediatr 12:317-324, 2013

12. Kobata H, Kondo A, Iwasaki K: Cerebellopontine angle epidermoids presenting with cranial nerve hyperactive dysfunction: pathogenesis and long-term surgical results in 30 patients. Neurosurgery 50:276-286, 2002

13. Lunardi P, Missori P, Gagliardi FM, Fortuna A: Long-term results of the surgical treatment of spinal dermoid and epidermoid tumors. Neurosurgery 25:860-864, 1989

14. Miller ME, Mastrodimos B, Cueva RA: Hearing preservation in management of epidermoids of the cerebellopontine angle: CPA epidermoids and hearing preservation. Otol Neurotol 33:1599-1603, 2012

15. Mohanty A, Venkatrama SK, Rao BR, Chandramouli BA, Jayakumar PN, Das BS: Experience with cerebellopontine angle epidermoids. Neurosurgery 40:24-30, 1997

16. Nagasawa D, Yew A, Safaee M, Fong B, Gopen Q, Parsa AT, et al: Clinical characteristics and diagnostic imaging of epidermoid tumors. J Clin Neurosci 18:1158-1162, 2011

17. Osborn AG, Preece MT: Intracranial cysts: radiologicpathologic correlation and imaging approach. Radiology 239:650-664, 2006

18. Rutherford SA, Leach PA, King AT: Early recurrence of an intracranial epidermoid cyst due to low-grade infection: case report. Skull Base 16:109-116, 2006

19. Sabin HI, Bordi LT, Symon L: Epidermoid cysts and cholesterol granulomas centered on the posterior fossa: twenty years of diagnosis and management. Neurosurgery 21:798805,1987

20. Samii M, Tatagiba M, Piquer J, Carvalho GA: Surgical treatment of epidermoid cysts of the cerebellopontine angle. $\mathbf{J}$ Neurosurg 84:14-19, 1996

21. Schiefer TK, Link MJ: Epidermoids of the cerebellopontine angle: a 20-year experience. Surg Neurol 70:584-590, 2008

22. Schwartz JF, Balentine JD: Recurrent meningitis due to an intracranial epidermoid. Neurology 28:124-129, 1978

23. Thomalske G, Kienow-Rieg I, Mohr G: Critical observations concerning the diagnosis and clinical features in 100 cases of tumors of the cerebellopontine region. Adv Neurosurg 1:252-259, 1973

24. Ulrich J: Intracranial epidermoids: a study on their distribution and spread. J Neurosurg 21:1051-1058, 1964

25. Vion-Dury J, Vincentelli F, Jiddane M, Van Bunnen Y, Rumeau C, Grisoli F, et al: MR imaging of epidermoid cysts. Neuroradiology 29:333-338, 1987

26. Winn HR (ed): Youmans Neurological Surgery, ed 6. Philadelphia: Elsevier-Saunders, 2011

27. Yaşargil MG, Abernathey CD, Sarioglu AC: Microneurosurgical treatment of intracranial dermoid and epidermoid tumors. Neurosurgery 24:561-567, 1989

28. Zhou LF: Intracranial epidermoid tumours: thirty-seven years of diagnosis and treatment. Br J Neurosurg 4:211216, 1990

\section{Disclosure}

The authors report no conflict of interest concerning the materials or methods used in this study or the findings specified in this paper.

\section{Author Contributions}

Conception and design: all authors. Acquisition of data: Guan, Hollon, Bentley. Analysis and interpretation of data: all authors. Drafting the article: Guan, Hollon, Bentley. Critically revising the article: all authors. Reviewed submitted version of manuscript: all authors. Approved the final version of the manuscript on behalf of all authors: Garton. Administrative/technical/material support: Garton. Study supervision: Garton.

\section{Correspondence}

Hugh J. L. Garton, Department of Neurosurgery, University of Michigan, Taubman Health Care Center, Rm. 3552, 1500 E. Medical Center Dr., Ann Arbor, MI 48109-5338. email: hgarton@med.umich.edu. 